\title{
Recent clinical investigations in migraine pain
}

\author{
Vincenzo Pizza ${ }^{1}$, Walter Milano ${ }^{2}$ and Anna Capasso ${ }^{3}$ \\ ${ }^{1}$ Neurophysiopathology Service, S. Luca Hospital, Vallo della Lucania (SA), Italy \\ ${ }^{2}$ Mental Health Unit District 24 ASL Napoli 1 Center, Italy \\ ${ }^{3}$ Department of Pharmacy, University of Salerno, Italy
}

\begin{abstract}
Migraine is a neuro-vascular syndrome characterised by headache attacks associated with photophobia, phonophobia, nausea and vomiting. Although the exact etiology of migraine is unknown, several theories have been proposed. The vascular theory attributes migraines to an initial intracranial arterial vasoconstriction resulting in reduced blood flow to the visual cortex, followed by a period of extra-cranial vasodilation. Modern imaging techniques have shown that during a common migraine attack there are in fact only minor changes in cerebral blood flow, and the proposed initial vasoconstrictive phase may actually last much longer than the aura. It has also been hypothesised that migraine sufferers have an inherent vasomotor instability and are more susceptible to the vasodilatory effects of certain physical and chemical agents. Migraine can be, according to our present knowledge, primary or direct, an invalidating disturbance largely spread ( $>12 \%)$ in the world population, having the form of an autonomous disease, as well secondary or indirect (2-3\%), in this last case being only a pathologic symptom of different etiologies. This review is centered on recent clinical investigations to reduce and/or to control migraine pain.
\end{abstract}

\section{Introduction}

The pathophysiology of migraine is not completely understood and continues to be investigated. Neuronal components are relevant in migraine pathophysiology: there could be a generalized interictal abnormal excitability of the cerebral cortex in migraine, possibly favoring the occurrence of spreading depression with consequent activation of the trigeminal system. The complexity of interactions taking place in the sensory neuronal network with the mediation of all different neurotransmitters involved gives the measure of the extreme difficulty connected with the knowledge of migraine pathogenesis and in particular of its cardinal sign, namely the pain, in spite of the very significant scientific contributions of these last years, from which the problem has received an enormous broadening and enlightenment. Many theories have been formulated in these last sixty years about the pathogenesis of migraine and other forms of primary headache, but the problem is still far to be fully clarified [1-3]. this review is concerned with the nature, type and proposed mechanisms of migraine pain as well as novel therapeutic applications.

The somatic sensitivity, which contributes to the preservation of physical integrity and homeostatic balance, depends upon the activation of sensitive receptors and relative nervous endings which are showing a different structural complexity and threshold and are distributed into cutaneous and visceral tissues as muscles, vessels and splanchnic organs. They convert physical and chemical stimuli into a painful afferent message, transmitted under the form of an action potential.

Primary sensory neurons, whose cellular bodies are located in the ganglia of dorsal spinal roots, forward to the central nervous system the action potential produced at the receptor site and relative nervous endings, being able to establish synaptic connections with second order neurons and, through them, with higher nervous centres, like thalamus and cerebral cortex [1-3].

The action potential conduction varies considerably in dependence of the afferent fibres dimension and myelinization. Teguments and joints contain myelinated sensory afferent fibres of middle diameter able to transport the stimulus with a relative rapidity (A-beta fibres), while free afferent endings and those coming from receptors activated by heat and other physical or chemical stimuli reach the spinal cord with thin fibres, poor of myelin (A-delta fibres) or without myelin (C fibres, defined polymodal).

The distribution of nociceptors is not uniform in the human body. They are present in large number at superficial levels (skin, cornea, tympanum) and in other areas, as dura mater, leptomeninges and vascular walls. From Gasser ganglion take origin nociceptive fibres directed to dura mater's vessels which play a prominent role in migraine pathogenesis. Actually their electrical stimulation produces, in animal experimental models, the release of neuropeptides as CGRP and substance $P$, which seem to be strongly involved in the induction of migraine pain; in the man, neurokinin $A$ can be considered the equivalent of substance $P$ in the rat.

The main function of nociceptors is to evidence the presence of noxious stimuli which, when applied for a sufficient time, are able to injure tissues by them innervated. Nociceptors do not show any spontaneous activity and in basal conditions possess an elevated threshold toward heat and mechanical stimuli. A-delta and C fibres increase significantly their sensitivity when are chemically sensitized by the endogenous substances released during the inflammatory process.

The sensitization is a peculiar function of nociceptors, consisting in a reduced threshold and an increased response to repeated or prolonged stimulations, as it happens for traumatic or inflammatory

Correspondence to: Anna Capasso, Department of Pharmacy, University of Salerno, 84084-Fisciano, Italy, E-mail: annacap@unisa.it

Key words: migraine, neurotransmitters, novel therapeutic applications, pain

Received: November 10, 2017; Accepted: December 14, 2017; Published: December 18,2017 
events. This phenomenon is due to chemical agents released at the tissue level following a cellular injury produced by noxious agents (Table 1$)$. Hydrogen $(\mathrm{H}+$ ) or potassium $(\mathrm{K}+)$ ions, bradykinin, histamine and serotonin, liberated by inflamed tissues, activate nociceptors with different mechanisms. The serotonin receptors stimulation provokes the opening of specific ionic channels. Bradykinin, through its receptors B2, induces the synthesis, mediated by the enzyme phospholipase C, of the intracellular second messenger inositoltriphosphate (IP3) and of diacilgycerol $(D G)$. Proteinkinase $C$, activated by $D G$, increases the conductance for $\mathrm{Na}+$ and $\mathrm{Ca}++$, so eliciting the genesis of the action potential and the consequential release of neuropeptides CGRP, substance $P$ and neurokinin A. Peripheral endings of sensory nociceptive neurons can exert also efferent functions. A subgroup of them, sensitive to the excitatory and desensitizing activities of capsaicin, which are able to synthesize neuropeptides in their cellular bodies, then transported to central and peripheral axonal endings [1-3]. This phenomenon is produced at the peripheral endings level also by the low $\mathrm{pH}$ present in the inflamed tissues because of the high content of protons, with consequent antidromic reflexes and release of neuropeptides CGRP and substance $P$, able to induce vasodilatation and increase vascular permeability in the proximity of the same endings, as well as passage in the interstitial spaces of kininogen, the precursor of bradykinin. The following synthesis of bradykinin brings to a further amplification of this vicious circle contributing in this way to raise the activation of nociceptors either directly or indirectly, through the stimulation of the synthesis and release of histamine, serotonin and prostaglandins, specially $P G E 2$. While the first two substances excite directly polymodal nociceptors, $P G E 2$, produced by the enzymatic activity of cyclooxygenase on its substrate arachidonic acid in mast cells, macrophages and other inflammatory cells at the level of injured tissues, induces hyperalgesia, mostly through a nociceptor sensitization. The result of this sequence of events is the induction of vasodilatation associated to increased vascular permeability and moreover to the stimulation of afferent endings of sensory capsaicin-sensitive neurons, with increase of intracellular AMPc levels and consequent activation of proteinkinase $A$, enzyme able to phosphorilate both voltage-dependent $\mathrm{Ca}++$ channels and tetradotoxin-resistant $\mathrm{Na}+$ channels.

These last two events, in turn, decrease the action potential threshold facilitating the activation of nociceptive fibres. The mechanism of action of aspirin and other NSAIDs, employed also in the therapy of migraine pain, is based on the inhibition of $C O X-1$ and $C O X-2$ and consecutive reduction of prostaglandin synthesis.

This review is centered on novel therapeutic applications to reduce and or to control migraine pain.

\section{Recent clinical investigations in migraine pain}

Lamey et al [4] report their study relates to a novel biochemical marker associated with migraine and a method of predicting forthcoming migraine attacks. The study also relates to a novel vasodilatory agent

Table 1. Substances released during inflammation able to activate or sensitize nociceptors

\begin{tabular}{|c|c|c|}
\hline SUBSTANCE & SOURCE & $\begin{array}{c}\text { ACTIVITY ON PRIMARY } \\
\text { AFFERENT FIBRES }\end{array}$ \\
\hline $\mathrm{K}+$ or $\mathrm{H}+$ & Injured cells & Activation \\
\hline Serotonin & Platelets & Activation \\
\hline Bradykinin & Plasmatic kyninogen & Activation \\
\hline Histamine & Mast cells & Activation \\
\hline Prostaglandins & Injured cells, Macrophages & Sensitization \\
\hline $\begin{array}{c}\text { Substance } \mathrm{P} \text { or } \\
\text { Neurokinin A }\end{array}$ & Primary afferent fibres & Sensitization \\
\hline
\end{tabular}

and to the use of a peptide to develop an antimigraine therapy. In a first aspect the present the study aims to provide a product to predict the onset of migraine. The study provides a method of predicting the onset of migraine attack, through the detection of elevated levels of salivary peptide Cystatin SN. Following the establishment of a patient's normal Cystatin levels, variations of this level can serve as an indication of an impending attack of migraine. According to the present study there is provided a method for predicting potential migraine attacks, the method comprising the steps of establishing a normal level of Cystatin SN for an individual and subsequently testing for variations thereof wherein elevated levels of Cystatin SN indicate the on set of a migraine attack. Elevated levels of Cystatin SN will preferably be at least three time the normal levels. Prediction of a migraine attack will enable an individual to commence treatment of the attack before onset therefore minimising any ill-effects or to plan ahead accordingly [4]. The study comprises the use of the amino acid sequence of Cystatin SN in the elucidation of the or a nucleic acid sequence in the development of a test for elevated expression of Cystatin SN. The The study provides such a test for detection of levels of expression of the Cystatin SN gene [4]. Lamey et al [4] have found that levels of Cystatin SN are about ten times higher in migraineurs than non-migraineurs and levels rose markedly in the 24 hours before a migraine attack. As this molecule is intimately linked with migraine attack, it can be concluded that its release as a result of tooth clenching may be the main factor responsible for attacks of migraine. Therefore, the present study provides a novel therapy for use in migraine management or prevention considering that the substance Cystatin $\mathrm{SN}$ is intimately linked to attacks of migraine [4]. This offers the opportunity to investigate the mechanism by which Cystatin SN causes attacks of migraine and therefor offers new drug possibilities as an agent for developing a drug against Cystatin SN or its receptor or increasing drug metabolism or excretion or allowing current drug therapies, some of which are not presently indicated for use in the treatment of migraine, to be given at an earlier stage when they perhaps would be effective. As there are no current reliable biochemical markers for migraine and there is some doubt as to the mechanism by which even acute migraine drug therapy is effective and as such the present invention in conjunction with the identification of the Cystatin $\mathrm{SN}$ molecule offers a different mechanism to prevent migraine attack [4]. The present study thus provides the use of a Cystatin molecule in developing a treatment for migraine wherein the treatment is based on an antagonist of cystatin.

Chissoe study [5] is related to the identification of genes that are associated with migraine and to screening methods to identify chemical compounds that act on those targets for the treatment of migraine or its associated pathologies. The purpose of the present study was to identify genes coding for tractable targets that are associated with migraine, to develop screening methods to identify compounds that act upon such targets, and to develop such compounds as medicines to treat migraine and its associated pathologies. Family and twin studies indicate that genetic factors are involved in the aetiology of migraine. A first aspect of the present study [5] is a method for screening small molecule compounds for use in treating migraine, by screening a test compound against a target selected from the group consisting of gene products of the genes APOE, GNAL, NEDD4L, PDIP, TPCN1, TRPM8, ADRA1B, P2RX4, TAAR2, TAAR3, USP 11, CHRNA5, RAB5A, DPP8, F2RL1, FZD5, PTGER1, SPI, ALOX5, CMTM8, DCBLD2, DPYS, IKBKB, OVCH1, PDE4DIP, PPM1G, PYY2, RYR1, BRD2, CAD, F2RL2, NCOA3, ADORA2B, BMX, CHRNA3/CHRNB4, F2R, GRIK5, ITGB4, MAPK10, NPEPL1, PTGIS, UCN2, and WASF1. Activity against said target indicates the test compound has potential 
use in treating migraine. The present study tested genes that encode for potential tractable targets to identify genes that are associated with the occurrence of migraine and to provide methods for screening to identify compounds with potential therapeutic effects in migraine [5]. An aspect of the present study is a method for screening small molecule compounds for use in treating migraine, by screening a test compound against a target selected from the group consisting of proteins encoded by the genes APOE, GNAL, NEDD4L, PDIP, TPCN1, TRPM8, ADRA1B, P2RX4, TAAR2, TAAR3, USP1, CHRNA5, RAB5A, DPP8, F2RL1, FZD5, PTGER1, SPI, ALOX5, CMTM8, DCBLD2, DPYS, IKBKB, OVCH1, PDE4DIP, PPM1G, PYY2, RYR1, BRD2, CAD, F2RL2, NCOA3, ADORA2B, BMX, CHRNA3/CHRNB4, F2R, GRIK5, ITGB4, MAPK10, NPEPL1, PTGIS, UCN2, and WASF1. Activity against said target indicates the test compound has potential use in treating migraine. Activity may be enhancing (increasing) the biological activity of the gene product, or diminishing (decreasing) the biological activity of the gene product [5].

Frants et al [6] report that family, twin and population-based studies suggest that genetic factors are involved in migraine, most likely as part of a multifactorial mechanism. The complex genetics has hampered identification of candidate genes for migraine. Familial Hemiplegic migraine (FHM) is a rare, autosomal dominant, subtype of migraine with aura, associated with ictal hemiparesis and, in some families cerebellar atrophy. Otherwise, the symptoms of the headache and aura phase of FHM and "normal" migraine attacks are very similar and both types of attacks may alternate within subject and co-occur within families. FHM is thus part of the migraine spectrum and can be used as a model to study the complex genetics of the more commonforms of migraine [6]. Since FHM is part of the migraine spectrum, thus be used to study the genetic factors and biological mechanisms that are related to various episodic neurological disorders such as FHM, EA-2, common migraine and others such as epilepsy. The present study [6] provides access to and methods to study the genetic factors and biological mechanisms that are related to various episodic neurological disorders such as FHM leading to migraine. The invention provides cells or animals in which changes such as deletions or mutations in said gene have been introduced by recombinant nucleic acid techniques. All such cells or animals provided by the invention can be used to study the pathophysiology of FHM, EA-2, migraine or other neurological disorders associated with cation channel dysfunction, for example to test or develop specific medication for the treatment of said disorders [6]. The study also provides proteins or peptides encoded by said genes, or fragments thereof, related with cation channel dysfunction, and detection of such proteins or peptides by antibodies directed against said proteins or peptides. Such antibodies can be of natural or synthetic origin, and can be produced by methods known in the art. Such proteins and antibodies and detection methods can be used to further in vitro or in vivo studies towards the pathophysiology of FHM, EA-2, migraine or other neurological disorders associated with cation channel dysfunction, in addition such proteins, antibodies and detection methods can also be used to diagnose or identify such disorders in patients or in experimental animals [6].

Lesniewski et al. study [7] relates to diagnostic assays useful with endothelin receptor antagonist therapy, and in particular relates to measurement of certain biomarkers that allow identification of patients eligible to receive endothelin receptor antagonist therapy and that permit monitoring of patient response to such therapy. The study has significant capability to provide improved selection of patients for Endothelin Receptor Antagonist therapy. The assessment of these biomarkers with the invention also allows tracking of individual patient response to the therapy. The inventive assays have utility with any ETRA therapy, including treatment of cancer, coronary angina, cerebral vasospasm, acute and chronic renal failure, gastric ulceration, cyclosporin-induced nephrotoxocity, endotoxin-induced toxicity, asthma, LPL-related lipoprotein disorders, other proliferative diseases, acute or chronic pulmonary hypertension, platelet aggregation, thrombosis, IL-2 mediated cardiotoxicity, colitis, vascular permeability disorders, ischemia-reperfusion injury, Raynaud's disease and migraine [7].

Sudilovsky et al, study [8], provided for inhibiting onset of or treating migraine headaches wherein a therapeutically effective amount of an angiotensin converting enzyme inhibitor alone or in combination with a calcium channel blocker is systemically, such as orally or parenterally, administered over a prolonged period, whereby frequency and intensity of migraine headaches are significantly reduced. In carrying out the method of the present invention, the angiotensin converting enzyme inhibitor alone or in combination with the calcium channel blocker may be administered to mammalian species, such as monkeys, dogs, cats, rats and humans, and as such may be incorporated in a conventional systemic dosage form, such as a tablet, capsule, elixir or injectable. The above dosage forms will also include the necessary carrier material, excipient, lubricant, buffer, antibacterial, bulking agent (such as mannitol), anti-oxidants (ascorbic acid of sodium bisulfite) or the like. Oral dosage forms are preferred, although parenteral forms such as intramuscular, intraperitoneal, or intravenous are quite satisfactory as well [8]. The dose administered must be carefully adjusted according to age, weight and condition of the patient, as well as the route of administration, dosage form and regimen and the desired result. The formulations as described above will be administered for a prolonged period, that is, for as long as the potential for onset of a migraine headache remains or the symptoms of a migraine headache continue. Sustained release forms of such formulations which may provide such amounts biweekly, weekly, monthly and the like may also be employed. A dosing period of at least two weeks and preferably at least 4 to 6 weeks are required to achieve minimal benefit [8].

Barlow et al study [9] is related to methods for treating diseases and conditions of the central and peripheral nervous system by stimulating or increasing neurogenesis via modulation of angiotensin activity. The study includes methods based on the application of an agent which modulates angiotensin action to stimulate or activate the formation of new nerve cells. Disclosed herein are methods for the prophylaxis and treatment of diseases, conditions and injuries of the central and peripheral nervous systems by stimulating or increasing neurogenesis. Aspects of the invention include increasing neurogenesis in cases of a disease, disorder, or condition of the nervous system. Embodiments of the invention include methods of treating a neurodegenerative disorder, neurological trauma including brain or central nervous system trauma and/or recovery therefrom, depression, anxiety, psychosis, learning and memory disorders, and ischemia of the central and/or peripheral nervous systems [9]. Also disclosed are methods for preparing a population of neural stem cells suitable for transplantation, comprising culturing a population of neural stem cells (NSCs) in vitro, and contacting the cultured neural stem cells with at least one modulator of angiotensin activity. In some embodiments, the stem cells are prepared and then transferred to a recipient host animal or human. Non-limiting examples of preparation include 1) contact with a modulator until the cells have undergone neurogenesis, 
such as that which is detectable by visual inspection, marker, or cell counting, or 2) contact with a modulator until the cells have been sufficiently stimulated or induced toward or into neurogenesis. The cells prepared in such a non-limiting manner may be transplanted to a subject, optionally with simultaneous, nearly simultaneous, or subsequent administration of a neurogenic agent, or a modulator of angiotensin activity to the subject. While the neural stem cells may be in the form of an in vitro culture or cell line, in other embodiments, the cells may be part of a tissue which is subsequently transplanted into a subject, based upon the cell morphology [9]. In yet another aspect, the study includes methods of stimulating or increasing neurogenesis in a subject by administering a modulator of angiotensin activity. In some embodiments, the neurogenesis occurs in combination with the stimulation of angiogenesis which provides new cells with access to the circulatory system [9].

\section{Conclusions}

The complexity of interactions taking place in the sensory neuronal network with the mediation of all different neurotransmitters involved gives the measure of the extreme difficulty connected with the knowledge of primary headache pathogenesis and in particular of its cardinal sign, namely the pain, in spite of the very significant scientific contributions of these last years, from which the problem has received an enormous broadening and enlightenment.

Many theories have been formulated in these last sixty years about the pathogenesis of migraine and other forms of primary headache, but the problem is still far to be fully clarified.

The first theory, conceived by Wolff in 1948 [10], is build up on a vascular basis, considering three main aspects: a) during the migraine attack extra-cranial vessels dilate and are throbbing in a large percent of patients; b) the stimulation of intra-cranial vessels provokes an ipsilateral headache; c) vasoconstrictor drugs, like ergot derivatives, show a curative effect, while vasodilators, like nitrates, may induce an attack. On this observations he hypothesized that an intra-cranial vasoconstriction could be responsible of the migraine aura and of the following hyperemic reaction associated to local vasodilatation, with consecutive activation of perivascular nociceptive endings. Ten years later Heyck [11] completed the theory adding the concept that during the attack arterio-venous anastomoses were closed, explaining in this way the decreased oxygen extraction observed in the symptomatic side.

Successive theories were underlining the importance of vasoactive and neuroactive substances (as plasmatic kinins, endorphins, serotonin, histamine, fatty acids, adenosine, prostaglandins, NO and endothelin-1), released in perivascular areas, to which could be attributed the responsibility of the neurogenic inflammation and its symptoms [12-15].

The already mentioned theory of Moskowitz [14] was synthesizing in an appreciable way all theories till now exposed, with the idea of the neurogenic inflammation in the trigemino-vascular area and the integration in the cerebral cortex of the painful information.

Another possibility to interpret the headache pain is proposed on the basis of a depression of the cortical electrical activity similar to the spreading depression observed in animal experimental models by Leao longtime ago (16) and consisting in the production of cortical hyperexcitation waves followed by the electrical activity suppression after chemical or mechanic stimuli, this last progressing by contiguity within the cerebral structures in posterior-anterior direction, at a speed of 2-5 $\mathrm{mm} / \mathrm{min}$ [17]. The spreading depression can be considered, according to Olesen [18], the pathophysiological substrate of the migraine aura, during which it has been observed with the technique of xenon 133 a decreased cerebral blood perfusion specially in the occipital area, lasting about an hour. These data have been validated by means of magneto-EEG, SPECT, MRI and PET studies [19], specially for the migraine with aura and, at least partially, also for the migraine without aura. Mitochondrial alterations and a decrease in $\mathrm{Mg}++$ blood levels have also been associated to migraine in neuroimaging investigations [20].

Lance [21], Diener and May [22], and Welch [23] have proposed to consider the participation in the migraine pathogenesis of orbital, frontal and limbic cortical areas and brainstem structures specialized in controlling pain mechanisms, like trigeminal nucleus caudalis, locus coeruleus, raphe nuccleus dorsalis, PAG and area postrema, interacting with several neurotransmitters as noradrenaline, serotonin, dopamine and others, which could be "migraine generators".

About the cluster headache, among other theories, it seems worthy of interest the immunological theory, dealing mostly with interleukins and cytokines receptors and involving the complex relationships between neuro-psychic, endocrine and immunological systems [24], as it is demonstrated by the reduced response of cortisol to metachlorophenyl-piperazine [25]. During the attack there is the activation of the hypothalamic grey ipsilateral to the side affected [26].

As far as tension migraine episodic and chronic, is concerned, it is not clear at all whether its pathogenesis is similar or different, and in what extent, in respect to that of migraine, but many resemblances have been recognised. The influence of stress and of protracted muscular contraction has been largely investigated as well as immunological implications, specially in the autoimmune sense [27]. It is necessary to assess the importance of genetic factors in the pathogenesis of migraine and migraine pain $[28,29]$.

In consideration of the complex migraine patohogenesis and frequent comorbidities, involving many receptorial functions and related metabolic pathways, number of drugs have been used in the prophylactic therapy and among them an increasing credit has in these last years acquired the approach based on minerals, vitamins, coenzymes and other nutrients, which can be associated to all other therapeutic strategies, exerting with them favourable synergistic activities.

Finally, it can not be omitted that the knowledge of migraine pain pathophysiology has tremendously improved because of the advances realised by clinical pharmacology studies, from which further and ever more important insights in the field likely are going to be reached in a next future.

\section{References}

1. Headache Classification Subcommittee of the International Headache Society (2004) The international classification of headache disorders.2nd. Oxford: Blackwell Publishing

2. Waeber C, Moskowitz MA (2003) Therapeutic implications of central and peripheral neurologic mechanisms in migraine. Neurology 61: S9-20. [Crossref]

3. Welch KM, D'Andrea G, Tepley N, Barkley G, Ramadan NM (1990)The concept of migraine as a state of central neuronal hyperexcitability. Neurol Clin 8: 817-828

4. Lamey S, Philip J, Lundy FT, Shaw M (2008) Migraine and vasodilation treatment, United States Patent.

5. Chissoe S (2008) Genes Associated with Migraine, United States Patent Application 0108078

6. Frants A, Rune IE, Michel D, Terwindt D, Gisela M, Ophoff, RA (2005) Gene related to migraine in man, United States Patent Application. 
7. Lesniewski R, Semizarov D, Van S, Charles L (2008) Companion diagnostic assays for endothelin receptor antagonists United States Patent Application.

8. Sudilovsky S (1990) Abraham Method for inhibiting onset of or treating migraine headaches employing an ACE inhibitor United States Patent H000734.

9. Barlow C, Carter TA, Treuner K, Lorrain KI (2008) Neurogenesis By Modulating Angiotensin, United States Patent Application

10. Wolff HG (1993) Headache and other head pain. New York: Oxford University Press.

11. Ninck B (1970) Migraine and epilepsy. Eur Neurol 3: 168-178. [Crossref]

12. Sicuteri F, Fanciullacci M, Anselmi B (1963) Mast cells and their active substances: their role in the pathogenesis of migraine. Int Arch Allergy 7: 88-92.

13. Sicuteri F (1972) Headache as possible expression of deficiency of brain 5 hydroxytryptamine. Headache 12: 69-72.

14. Moskowitz MA (1992) Neurogenic versus vascular mechanisms of sumatriptan and ergot alkaloids in migraine. Trends Pharmacol Sci 13: 307-311. [Crossref]

15. Hoskin KL, Bulmer DC, Goadsby PJ (1999) Fos expression in the trigeminocervical complex of the cat after stimulation of the superior sagittal sinus is reduced by L-NAME. Neurosci Lett 266: 173-176. [Crossref]

16. Leao AA (1941) Spreading depression of activity in the cerebral cortex. Arch Neurol Psychiatry 46: 333-339.

17. Lauritzen M (1994) Pathophysiology of the migraine aura. The spreading depression theory. Brain 117: 199-210. [Crossref]

18. Olesen J, Friberg L, Olsen TS (1990) Timing and topography of cerebral blood flow, aura, and headache during migraine attacks. Ann Neurol 28: 791-798.

19. Cutrer FM, Sorensen AG, Weisskoff RM, Ostegaard L, Sanchez del Rio M, et al. (1998) Perfusion-weighted imaging defects during spontaneous migrainous aura. Ann Neurol 43: 25-31.
20. Lodi R, Iotti S, Cortelli P, Pierangeli G, Cevoli S, et al. (2001) Deficient energy metabolism is associated with low free magnesium in the brains of patients with migraine and cluster headache. Brain Res Bull 54: 437-441. [Crossref]

21. Gallai V, Sarchielli P, Coata G, Firenze C, Morucci P, Abritti G (1992) Serum and Salivary magnesium levels in migraine: results in a group of juvenile patients. Headache 32: 132-135.

22. Lance JW (1992) The pathophysiology of migraine: a tentative synthesis. Pathol Biol (Paris) 40: 355-360. [Crossref]

23. Diener HC, May A (1996) New aspects of migraine patho-. physiology: lessons learned from positron emission tomography. Curr Opin Neurol 9: 199-201.

24. Welch KMA, Cao Y, Aurora S, Wiggings G, Vikingstad EM (1998) MRI of the occipital cortex, red nucleus and substantia nigra during a aura of migraine. Neurology 51: 1465-1469.

25. Giacovazzo M, Martelletti P, Valeri M, Piazza A, Casciani CU (1986) A new immunological aspect of cluster headache: the increase of monocyte and NK cell populations. Headache 26: 134-136.

26. Waldenlind E, Bussone G (2000) In: The headaches. 2nd. Olesen J, Tfelt-Hansen P, Goadsby PJ, editors. Philadelphia: Lippincott-Williams and Wilkins pp. 687-95.

27. Leone M, Franzini A, Bussone G (2001) Stereotactic stimulation of posterior hypothalamic gray matter in a patient with intractable cluster, headache. $N$ Engl J Med 345: 1428-1429.

28. Sicuteri F (1976) Hypothesis: migraine, a central biochemical dysnociception Headache 16: 145-159. [Crossref]

29. Montagna P (2000) Molecular genetics of migraine headaches: a review. Cephalalgia 20: 3-14. [Crossref]

Copyright: (C2017 Pizza V. This is an open-access article distributed under the terms of the Creative Commons Attribution License, which permits unrestricted use, distribution, and reproduction in any medium, provided the original author and source are credited. 